\title{
Indication of quantum mechanical electron transport in human substantia nigra tissue from conductive atomic force microscopy analysis
}

Christopher J. Rourk, Dallas, TX

\begin{abstract}
Neuromelanin and ferritin in dopamine neurons of the substantia nigra have a distribution and density that is similar to disordered arrays of quantum dots in photovoltaic devices, which have been experimentally shown to conduct electric energy using quantum mechanical electron transport mechanisms. Conductive atomic force microscopy tests were performed on human substantia nigra tissue at room temperature, to determine whether evidence of electron transport is present. The test results presented here provide evidence of quantum mechanical electron transport from ferritin and neuromelanin at levels that that are sufficient to cause or contribute to generation of action potentials.
\end{abstract}

\section{Introduction}

It has been proposed that neuromelanin (which is present in neuromelanin organelles (NMOs)) and ferritin in the substantia nigra (SN) dopamine neurons and surrounding tissue form a disordered array, and could function in a manner similar to quantum dots in solar photovoltaic devices (Rourk, 2018). In particular, ferritin has been experimentally shown to exhibit electron transport capabilities (Axford and Davis, 2007; Rakshit and Mukhopadhyay, 2012; Bostick et al., 2018; Kumar et al., 2016). There are also similarities between the distribution of those biomolecules in SN tissue and disordered quantum dot structures that have been studied for use in semiconductor devices (Sun et al., 2008; Lazarenkova and Balandin, 2002). In general, these biomolecules form disordered arrays in dopamine neurons, which themselves are disposed in a concentrated area of the $\mathrm{SN}$, and it is possible that this disordered array of biomolecules could be used to conduct electrical energy between dopamine neurons in some manner, such as to assist with the generation of action potentials, neuron pacemaker activity coordination or other cellular functions (Rourk, 2018). However, no measurements have ever been conducted to validate the hypothesis that quantum electron transport is possible in neural tissue. 
In order to obtain experimental data that would provide additional evidence in regards to that hypothesis, conductive atomic force microscopy (c-AFM) measurements were performed on normal (non-diseased) human SN tissue. C-AFM testing exposes a sample to electric fields that are sufficient to generate excitons, and has been used on materials other than neural tissue to generate excitons and electron transport. The equipment used to perform C-AFM testing is capable of measuring current levels in the fA range, for samples as small as $2 \mu \mathrm{m} \times 2 \mu \mathrm{m}$.

The experimental procedures used to prepare the tissues for c-AFM testing and for performing the c-AFM testing are discussed below. Four tests were performed that yielded useful results. These tests indicate that electron transport may be occurring in these tissue samples at a measurable level. These experiments advance our understanding of whether it is possible for quantum mechanical electron transport to be present in neural tissue by showing that levels of electron transport are present during c-AFM testing that would potentially be sufficient to create or contribute to the creation of an action potential.

\section{Experimental Procedures}

The SN tissue samples were obtained from AMSBIO of Cambridge, MA, and were fixed in $10 \%$ buffered neutral formalin and processed for embedding into low temperature melting paraffin. The tissue samples were then cut at a nominal thickness of $5 \mu \mathrm{m}$ and mounted on 1) positively charged glass slides, and 2) glass slides wrapped in aluminum foil, to provide electrical contact for c-AFM testing. The tissue samples were maintained at room temperature for shortterm storage and at $-4^{\circ} \mathrm{C}$ for long-term storage in a sealed bag with desiccant, and were tested within 1 year of the date of purchase. Optical microscope data from one of the tissue samples on a glass slide was compared to optical microscope data from the tissue samples on foil, and while the tissue samples on foil were more difficult to inspect for NMOs due to the absence of backlighting, the cellular structures associated with NMOs that were apparent from the tissue samples on foil also appeared in the tissue sample on the glass slide. These cellular structures were used to select areas for c-AFM testing.

Two tissue sample preparation procedures were used. For a first sample, the tissue was soaked in xylene twice for 5 minutes each time to remove wax, per AMSBIO recommendations. For a second sample, the tissue was soaked in xylene twice for 30 minutes after heating for 10

minutes at $65^{\circ} \mathrm{C}$, followed by successive ethanol soaks with increasing levels of water, to rehydrate the tissue. The second protocol was followed to ensure adequate wax removal after 
review of the first c-AFM test using the first protocol, and was used for the other three c-AFM tests.

The SN tissue samples were shipped directly from AMSBIO to EAG Laboratories of Sunnyvale, CA for c-AFM testing using a Dimension Icon AFM instrument from Bruker of Santa Barbara, California. C-AFM testing is conducted by using a micron-scale cantilever probe to tap along the surface of a specimen (there is also a non-tapping mode of c-AFM, but that was not used for these tests). The probe has a pyramidal-shaped tip that extends downwards for several microns, and has a tip with dimensions of approximately 5 to $10 \mathrm{~nm}$ radius, on a new tip. The probe is biased with a voltage, and three current data points are generated during tapping mode operation: 1) peak current, 2) cycle-averaged current, and 3) contact-averaged current. Peak current is the instantaneous current at the point of contact and coincides with the peak applied force. This peak current is not necessarily the maximum current, because the rise time is limited by the bandwidth of the current probe module and the impedance of the sample, which can cause a lag in the current response. The cycle-averaged current is the average current over one full tapping cycle and includes both the current measured while tip is in contact with the surface, and while it is off the surface, but the contact-averaged current is the average current only when the tip is in contact with the surface. For these tests, the probe made 512 measurements at a frequency of approximately $0.2 \mathrm{~Hz}$ for each linear scan (trace and retrace), and made 512 linear scans, for a total of 262,144 measurement locations per sample. In addition to the current measurements, the c-AFM probe also provides quantitative measurement of 1) height, 2) elasticity, 3) force, 4) deformation and 5) dissipation.

In addition to the measurements at each point, current-voltage curves can be generated at selected points as the applied voltages is varied from a maximum of $10 \mathrm{~V}$ to a minimum of $-10 \mathrm{~V}$. Those current-voltage curves are not discussed here, as they did not provide evidence that was as useful as the arrays of data presented here. It is also noted that two different current amplifiers were used to measure the contact, peak and average currents, a first one having a sensitivity of 1 nano-ampere per volt (which was used for the $76 \mu \mathrm{m} \times 76 \mu \mathrm{m}$ sample tests), and a second one having a sensitivity of 20 picoamperes per volt (which was used for the $2 \mu \mathrm{m}$ x $2 \mu \mathrm{m}$ sample test).

\section{Samples}

Four samples were tested:

1) Test One - short wax removal protocol, $76 \mu \mathrm{m} \times 76 \mu \mathrm{m}$ test area, cell-shaped area 
2) Test Two - long wax removal protocol, $76 \mu \mathrm{m} \times 76 \mu \mathrm{m}$ area, cell-shaped area

3) Test Three - long wax removal protocol, $2 \mu \mathrm{m} \times 2 \mu \mathrm{m}$ detail within Test Two area

4) Test Four - long wax removal protocol, $76 \mu \mathrm{m} \times 76 \mu \mathrm{m}$ area, no cell-shapes (dark tissue appearance)

\section{Test results}

The Test One c-AFM results provided numerous positive peak current, cycle-averaged current, and contact-averaged current indications at a bias voltage of $-10 \mathrm{~V}$, as well as numerous negative peak current, cycle-averaged current and contact-averaged current indications. The data from that test is not presented here. Based on those results, the amount of wax removal was evaluated, and it was determined that a more thorough wax removal protocol than the one that was recommended by AMSBIO would likely improve the ability to perform current measurements. A suitable protocol was located and used for the remaining tests.

Figure 1A-A is an optical photograph of the Test Two SN tissue sample disposed on foil. Because of the absence of backlighting, neuromelanin cannot be seen, but the size (approximately $25 \mu \mathrm{m})$ and oval shape of five areas in the center of the image are similar to the size and shape of the dopamine neurons and nuclei from stained tissue photographs that can be seen in the literature, such as Halliday et al., 2005. Darker regions can be seen that surround cell nuclei that contain neuromelanin and ferritin (Tribl et al., 2009). It is also noted that the apparent spacing between cell bodies in Figure 1A is less than $10 \mu \mathrm{m}$, which is substantially closer than the assumed spacing of $100 \mu \mathrm{m}$ in Rourk, 2018.

Figure 1A-B is a two-dimensional image of AFM height measurements for the area that is contained within the square shown in Figure 1A-A. An autoscale generated by the Bruker data mapping software ranges from $-3.1 \mu \mathrm{m}$ to $3.1 \mu \mathrm{m}$, which correlates to the nominal $5 \mu \mathrm{m}$ thickness of the SN tissue samples. A histogram of this height data is shown in Figure 1B-A. The darker, lower-thickness areas generally have a variable height, when viewed at a higher resolution. Based on the thickness data and the assumptions that the foil would be relatively flat over the $76 \mu \mathrm{m} x$ $76 \mu \mathrm{m}$ sample and that the foil is at or below the lowest height measurement (approximately -2.4 $\mu \mathrm{m}$ ), areas above that lowest height level are either 1) tissue structure, or 2) detritus that would have been suspended in intracellular or extracellular fluid but which remained after fluids were replaced by formalin and/or wax. The thinnest areas likely correspond to the detritus from material that would have been suspended in intracellular fluid, based on the spacing, shape and size of those 
areas. While these areas would likely contain the highest density of neuromelanin and ferritin from the cell body, they would not exhibit the spacing that would be seen in a living cell, and would be expected to have a negative impact on electron transport current measurements, due to the importance of spacing in the generation of electron transport.

Figure $1 \mathrm{~A}-\mathrm{C}$ is a two-dimensional image of the contact-averaged current. The auto-scale shows a range of -1.7 to $2.5 \mathrm{nA}$, but the histogram data of Figure 1B-B shows that the contactaveraged current ranges from approximately $-20 \mathrm{nA}$ to $2 \mathrm{nA}$. The lowest contact-averaged current values are generally found in the thin areas that correspond to the intracellular detritus accumulations, which suggests that these areas have better electrical contact to the underlying foil layer.

Figure 1A-D is a two-dimensional image of the contact-averaged current. The auto-scale shows a range of -236 to $181 \mathrm{nA}$, but the histogram data of Figure 1B-D shows that the contactaveraged current includes a small number of data points that extend to approximately $-20 \mathrm{nA}$, and that most data points are between 0 and $2 \mathrm{nA}$.

Figures 1B-A through 1B-D are histograms of the height data, the peak contact current data, the contact-averaged current data and the cycle-averaged current data, respectively. Several observations can be made. First, the peak contact current data, the contact-averaged current data and the cycle-averaged current data vary significantly, and do not have the same distribution. This indicates that the current changes non-linearly and significantly over the measurement period for each contact. Second, the peak contact current data, the contact-averaged current data and the cycle-averaged current data are not linearly related to the thickness, which would be expected if the impedance between the c-AFM probe tip and the foil substrate was linear. Third, the peak contact current data, and the contact-averaged current data distributions have significant positive current components, which would not be expected from a $-10 \mathrm{~V}$ tip bias voltage. Fourth, it is noted that the majority of height measurements are substantially greater than the minimum, which indicates that the negative currents that are measured are not caused by direct contact with the foil substrate, but rather that the conductive cellular detritus layer does not have impedance characteristics that vary linearly with thickness. Finally, there are a large number of positive peak contact current and contact-averaged current measurement readings in the detritus field, which demonstrates that the components that are responsible for that current behavior are distributed 
throughout those regions, as well as throughout the thicker tissues that appear to maintain at least some of their structure.

The arrows shown in each of Figures 1A-B, 1A-C and 1A-D show the approximate location of the second test sample, which was a $2 \mu \mathrm{m} \times 2 \mu \mathrm{m}$ section from within the first sample region, at a point that corresponds to an area with one of the highest magnitude peak contact current readings. This location was chosen because the high levels of contact current should correspond to locations with a high concentration of ferritin and neuromelanin, but at a spacing of less than several hundred nanometers.

Figure 2A-A is a two-dimensional image of the contact-averaged current readings of the Test Three high-resolution $2 \mu \mathrm{m} \times 2 \mu \mathrm{m}$ section. Although the auto-scale shows a range of -3.4 to $8.2 \mathrm{pA}$, the histogram data shows that most contact-averaged current readings fall between -2 and $6 \mathrm{pA}$. Three round areas are identified with arrows that range from 200 to $400 \mathrm{~nm}$ in diameter, which corresponds to the size of NMOs that has been reported in the literature (Trible et al., 2009). In addition, sheet-like regions of high contact-averaged current appear to be associated with different tissue structures, as shown in the height data of Figure $2 \mathrm{~A}-\mathrm{C}$, but which do not necessarily correlate directly to those structures. The spatial resolution of the AFM height data is not accurate enough to determine whether these sheet-like structures include ferritin. The spacing between the NMOs and the sheet-like structures is within $400 \mathrm{~nm}$, and between sheet-like structures is within $100 \mathrm{~nm}$.

Figure 2A-B is a three-dimensional projection of the contact-averaged current readings of the high-resolution $2 \mu \mathrm{m} \times 2 \mu \mathrm{m}$ section. This figure more clearly shows the relative contactaveraged current differences between the NMOs and the sheet-like structures, and also shows that the highest contact-averaged currents are along the left-most side of the scan. These readings appear to be truncated at $8.2 \mathrm{pA}$, the maximum reading shown on the auto-scale.

Figure 2A-C is a three-dimensional projection of the height readings of the high-resolution $2 \mu \mathrm{m} \times 2 \mu \mathrm{m}$ section. This figure more clearly shows a lower thickness area on the left-most side of the scan, with areas of increasing thickness moving from left to right in the figure. The borders between regions where the thickness changes appear to be associated with the sheet-like contactaveraged current structures, but there is not a direct correlation between those two features at all points. It is also noted that the NMO structures are not associated with any surface structures, and 
appear to be associated with NMOs that are covered by a layer of material that may include portions of the cytoskeleton, lipids or other materials.

Figure 2A-D is a three-dimensional projection of adhesion readings of the high-resolution $2 \mu \mathrm{m} \times 2 \mu \mathrm{m}$ section. This figure more clearly shows spherical NMO structures based on the material property of adhesion, and also shows that the sheet-like areas of higher contact current appear to correlate more closely with areas having higher adhesion. While the interpretation of such adhesion readings is not addressed in this analysis, these adhesion readings appear to indicate the presence of different materials and the borders between those materials.

Figures 2B-A through 2B-D are histograms of the height data, contact-averaged current data, peak contact current data and cycle-averaged current data, respectively. Several observations can be made. First, the peak contact current data, the contact-averaged current data and the cycleaveraged current data vary significantly, and do not have the same distribution, as was the case with the Test Two data, also indicating that the current changes non-linearly and significantly over the measurement period for each contact. Second, the peak contact current data, the contactaveraged current data and the cycle-averaged current data are not linearly related to the thickness, as was the case with the Test Two data. Third, the peak contact current data, and the contactaveraged current data distributions have significant positive current components, as was the case with the test Two data. Fourth, it is noted that the majority of height measurements are substantially greater than the minimum, which indicates that the negative currents that are measured are not caused by direct contact with the foil substrate, but rather that the conductive cellular detritus layer does not have impedance characteristics that vary linearly with thickness. Finally, the peak contact current data and the contact-averaged current data include negative current values, but the cycle-averaged current data is predominantly positive. This is also the case for the Test Two data, but the histogram data covers a greater range, due to a number of negative cycle-averaged current data readings that comprise a very small percentage of the total number of readings.

Figure 3A-A is an optical photograph of the Test Four SN tissue sample disposed on foil. Because of the absence of backlighting, neuromelanin cannot be seen, but the darker area was investigated to determine whether it contained neuromelanin. There are no apparent dopamine neurons in the area that was tested. 
Figure $3 \mathrm{~A}-\mathrm{B}$ is a three-dimensional projection image of contact-averaged current measurements for the area that is contained within the square shown in Figure 1A-A. A histogram of this height data is shown in Figure 3B-B, which shows that these readings are very close to zero but the histogram data covers a greater range, due to a number of negative cycle-averaged current data readings that comprise a very small percentage of the total number of readings. A review of the data that was used to generate the histogram shows that greater than $90 \%$ of the readings are under $3 \mathrm{pA}$.

Figure 3A-C is a two-dimensional image of the height data. The auto-scale shows a range of -2.6 to $2.4 \mu \mathrm{m}$ relative to a middle point of 0 , but the histogram data of Figure 3B-A shows that the absolute height mostly ranges from approximately 3 to $7 \mu \mathrm{m}$. The height at the top of the foil layer is not known.

Figure 3A-D is a two-dimensional side image of the contact-averaged current. The autoscale shows a range of $-2.1 \mathrm{nA}$ to $421 \mathrm{pA}$, but the histogram data of Figure 1B-D shows that the contact-averaged current is primarily approximately zero.

Figures 3B-A through 1B-D are histograms of the height data, peak contact current data, the contact-averaged current data and the cycle-averaged current data, respectively. Several observations can be made. First, the peak contact current data, the contact-averaged current data and the cycle-averaged current data do not vary significantly, and have approximately the same distribution of almost 0 amperes. This indicates that the current does not change significantly over the measurement period for each contact. Second, the peak contact current data, the contactaveraged current data and the cycle-averaged current data are not linearly related to the thickness, which would be expected if the impedance between the c-AFM probe tip and the foil substrate was linear. Third, these current readings are unlike the current readings for the other three tested areas, and demonstrates that the typical current response for c-AFM testing of tissue samples is not substantial, and that the results observed in the other three areas are not typical of c-AFM testing of tissue samples that do not include high concentrations of ferritin and neuromelanin.

\section{Analysis}

There was a substantial difference between the currents measured for Test One, and the currents measured for Tests Two through Four. While the Test One results yielded a number of positive current readings, Tests Two and Three yielded a substantially greater number of positive current measurements, which indicates that wax removal is important to improve the ability to 
measure c-AFM currents. The second wax removal protocol was identified from a resource for tissue staining, and is beneficial for c-AFM testing of tissue, as it appears to remove additional wax layers that might be only several hundred nanometers in thickness. Due to the apparent absence in the literature of any reports of c-AFM testing on tissue, additional research in this area would be useful, not only on wax removal protocols for animal tissues for c-AFM testing, but also on c-AFM testing of other suspected biological quantum dot structures, such as the Fenna-Matthews-Olson (FMO) complex and Light Harvesting Complex II (LHCII).

While there are a number of references in the technical literature that discuss c-AFM measurements of quantum dots, these references are generally directed to measuring individual quantum dot currents, with a small number of additional individual quantum dots in the near vicinity that may have some impact on the current measurements, but which do not form large arrays of quantum dots that can generate electron transport currents (Zhang et al., 2009; Tanaka et al., 1999). Other studies involve measurements of conduction currents through monolayers of quantum dots (Wold et al., 2002; Casuso et al., 2007). While Zhang reports positive currents with applied negative bias voltages, none of these studies involved large-scale three dimensional devices that included thousands of quantum dots or more, or measurement of tissues with quantum dots disposed in the tissue. As such, there does not appear to be sufficient pre-existing literature to assist with confirming the interpretation of these test results.

The most significant observation from these tests is the generation of positive peak contact current data, contact-averaged current data and cycle-averaged current data with a negative applied bias. A bias voltage of $-10 \mathrm{~V}$ and a distance between a planar electrode and a foil ground plane of $5 \mu \mathrm{m}$, which is the nominal thickness of the tested SN tissue samples disposed on foil substrate, would result in an electric field magnitude of 2 million volts per meter. The sharp tip of the c-AFM probe creates a substantial increase in the electric field gradient, and the tissue thickness was variable over the test regions and often less than $5 \mu \mathrm{m}$ in thickness, which would further increase the electric field magnitude in areas near the tip. Electric fields at these magnitudes are high enough to cause ionization of molecular structures with low ionization energies. If such molecular structures exhibit quantum dot characteristics, the free electrons and the associated holes created by ionization could form excitons, and support the creation of coherent electrons and formation of electron transport. A large number of coherent electrons forming electron minibands or other electron transport mechanisms (such as electron 
hopping or tunneling) could generate an effective negative voltage that is greater in magnitude than the $-10 \mathrm{~V}$ bias of the c-AFM probe tip, and would explain the positive current flow into the c-AFM tip. In the absence of such electron transport, it would be expected that the electrons supplied from the $-10 \mathrm{~V}$ bias of the c-AFM probe tip would only create negative currents, which is what was observed in locations where the thickness of the sample was much lower.

The Bruker Dimension c-AFM system compensates for capacitive charging current, so such currents would not be a factor in any measurements. Regardless of whether the tissue impedance is mostly resistive or includes capacitive or even inductive impedance components (which is unlikely), capacitive and inductive circuit elements would still draw charging current at contact and should not be able to provide positive contact current to a negative biased probe. Furthermore, the absence of such currents in the Test Four sample demonstrates that the effect of such resistive, capacitive or inductive contact and peak current components are negligible in the Test Two and Test Three results.

The ability of the Bruker Dimension Icon to measure peak contact current data, contactaveraged current data and cycle-averaged current data provides some insight into the transient nature of these currents. The scan rate for these c-AFM measurements was approximately 0.2 $\mathrm{Hz}$, which results in a maximum time per contact of 200 microseconds, and given the transit time between contact points, the actual contact time was probably less than 40 microseconds (assuming that the contact time was $10 \%$ of the transit time between measurement points, which is conservative). The difference between the contact-averaged current, peak contact current and cycle-averaged current suggests that a high frequency current redistribution process is occurring at many measurement points, which would correlate to known electron coherence times in the hundreds of femtosecond range at room temperature for other biomolecular quantum dot structures such as FMO or LHCII. If a large number of ferritin and neuromelanin molecules are involved in the generation of excitons and coherent electron transport mechanisms, a complex high frequency current pattern would be expected.

For example, the measured peak contact currents of $2 \mathrm{nA}$ and associated cycle-averaged current in the range of $500 \mathrm{fA}$ suggests that the peak contact current transient had a duration of approximately $1 / 4000^{\text {th }}$ of the duration of the contact cycle, or 10 nanoseconds. Assuming that the mean lifetime of an exciton is 400 femtoseconds and electron coherence, that would allow 25,000 exciton coherence events to occur as part of the cycle-averaged current measurement. 
A sustained current of $2 \mathrm{nA}$ would result in the transfer of approximately $1.2010^{13}$ electrons per second, such that the number of electrons transferred over 10 nanoseconds would be approximately $10^{8}$ electrons. Assuming that each electron represents an exciton generated by a ferritin or neuromelanin molecule, that would suggest that the measured currents may have involved electron transfer from multiple adjacent dopamine neurons in the tissue samples, which may contain on the order of $1 \times 10^{6}$ ferritin cores in a $25 \mu \mathrm{m}$ diameter cell (Bertini et al., 2012). Further, assuming that action potentials in an average neuron can be generated by an influx of approximately $2 \times 10^{7}$ sodium ions, these c-AFM measurements indicate that a sufficient level of electron transport might be able to occur in the SN to be a factor in the generation of action potentials, although further research and a more rigorous analysis would be required to substantiate that hypothesis.

The correlation between the NMO-sized regions and the positive contact currents is also notable, and further suggests that neuromelanin has quantum dot properties, similar to those that have been observed for ferritin. The adhesion and elasticity readings further confirmed the correlation between the size and shape of those features and the size and shape of NMOs. None of these regions appeared to have an observable surface feature based on the AFM height data, and the NMOs may have been underneath some thickness of intervening tissue structure, a lipid layer or other materials. The similarity between the boundary layer effects at the edges of the second test area of the second sample could be explained by ferritin accumulations in these locations. The highest contact current peaks correspond to the thinnest region, which could include exposed ferritin molecules that were able to make physical contact with the c-AFM probe, whereas lower contact-averaged current regions may include ferritin molecules that have several hundred nanometers of covering tissue or material, which would limit currents as a function of electron tunneling or other quantum mechanical electron transport effects.

Another observation that can be made from these test results is that the presence of a wax coating appears to have an impact on the results. While the thickness of the remaining wax coating after the first and second wax removal protocols is not known, the second wax removal protocol included a substantially longer exposure to the wax solvent xylene after heating, and successive ethanol soaks. It is also possible that tissue hydration increased tissue conductivity or other electrical effects, although that should not have had any impact on the generation of positive contact-averaged current, peak contact current and cycle-averaged current. It would not 
be unexpected for a remaining thickness of several hundred nanometers of wax to be present after the first wax removal protocol, and for that layer to be removed by the second wax removal protocol, and a thickness of that amount of an intervening space could be sufficient to prevent the tip of the c-AFM probe from interacting with a disordered array of neuromelanin and ferritin to receive electron transport currents.

The distribution of positive contact-averaged current, peak contact current and cycleaveraged current in the second test location is consistent with estimates of the density of ferritin in $\mathrm{SN}$ tissue, and suggests that c-AFM probe measurements within several hundred nanometers are sufficient to measure currents from electron transport. Positive contact-averaged current, peak contact current and cycle-averaged current were also measured at numerous locations in the thinner region that appear to correspond to regions of intracellular fluid, and may also indicate that a wax layer of several hundred nanometers thickness or more is sufficient to prevent electron transport current to flow from the ferritin and neuromelanin into the c-AFM probe tip in these regions.

It is hypothesized that the measured electron transport currents could be used by SN neurons to coordinate retinotopically-mapped neural inputs to the $\mathrm{SN}$ with other corresponding retinoptopically-mapped neural inputs from cortical regions associated with image data processing and object recognition (Desimone et al., 2015). For example, this electron transport mechanism could explain the ability to identify objects within image data and to understand what those objects are, by integrating neural inputs into the SN from different regions of the cerebral cortex, which are also retinotopically organized (Erlikhman et al., 2016). The ability of coherent electrons to integrate these inputs into what is understood as the conscious experience suggests that coherent electrons are capable of separating the neural inputs into a large number of states, and of sharing these states with other coherent electrons in some manner, although additional work would be required to test that hypothesis. Controlled electrical or chemical stimulation of the SN synaptic connections could potentially be used to determine whether these inputs are determinative of the conscious experience.

\section{Conclusion}

These c-AFM tests appear to demonstrate highly non-linear behavior that correlates to electron transport in quantum dot arrays. The currents measured could be the result of electron transport levels that would be sufficient to influence or to potentially determine action potential 
generation. Additional testing of additional areas of tissue at different bias voltage could be performed to confirm these test results. 


\section{References}

Axford, D., Davis, J.J., 2007. Electron flux through apo-and holoferritin. Nanotechnology $18,145502$.

Bertini, I., Lalli, D., Mangani, S., Pozzi, C., Rosa, C., Theil, E.C., Turano, P., 2012. Structural insights into the ferroxidase site of ferritins from higher eukaryotes. J. Am. Chem. Soc. 134, 61696176.

Bostick, C.D., Mukhopadhyay, S., Pecht, I., Sheves, M., Cahen, D., Lederman, D., 2018.

Protein bioelectronics: a review of what we do and do not know. Rep. Prog. Phys. 81, 026601.

Casuso I., Fumagalli L., Samitier J., Padrós E., Reggiani L., Akimov V., Gomila G. (2007), Electron transport through supported biomembranes at the nanoscale by conductive atomic force microscopy, Nanotechnology 18:46.

Desimone K., Viviano J., Schneider K. (2015), Population Receptive Field Estimation Reveals New Retinotopic Maps In Human Subcortex, Journal Of Neuroscience 35 (27) 9836-9847

Erlikhman G, Gurariy G, Mruczek REB, Caplovitz GP (2016), The neural representation of objects formed through the spatiotemporal integration of visual transients. Neuroimage. 42:67-78.

Halliday G., Ophof A., et al. (2005), A-Synuclein Redistributes To Neuromelanin Lipid In The Substantia Nigra Early In Parkinson's Disease, Brain, 128:11, 2654-2664.

Kumar, K.S., Pasula, R.R., Lim, S., Nijhuis, C.A., 2016. Long range tunneling processes across ferritin-based junctions. Adv. Mater. 28, 1824-1830.

Lazarenkova, O.L., Balandin, A.A., 2002. Electron and phonon energy spectra in a three dimensional regimented quantum dot superlattice. Phys. Rev. B 66 245319-1-245319-9. 
Rakshit, T., Mukhopadhyay, R., 2012. Solid-state electron transport in Mn-, Co-, holo-, and $\mathrm{Cu}$-ferritins: force-induced modulation is inversely linked to the protein conductivity. J. Colloid Interface Sci. 388, 282-292.

Rourk C (2018), Ferritin and neuromelanin "quantum dot" array structures in dopamine neurons of the substantia nigra pars compacta and norepinephrine neurons of the locus coeruleus, Biosystems, 171:48-58.

Sun, K., Li, Y., Stroscio, M.A., Dutta, M., 2008. Miniband formation in superlattices of colloidal quantum dots and conductive polymers. ECS Trans. 6, 1-12.

Tanaka I, Kamiya I, Sakaki H (1999), Imaging and probing electronic properties of self-assembled InAs quantum dots by atomic force microscopy with conductive tip, Appl. Phys. Lett. 74, 844.

Tribl F, Asan E., et al. (2009), Identification of L-ferritin in neuromelanin granules of the human substantia nigra: A targeted proteomics approach. Molecular \& cellular proteomics MCP. 8. 18328.

Wold D., Haag R., Rampi M.A., Frisbie, C.D (2002), Distance dependence of electron tunneling through self-assembled monolayers measured by conducting probe atomic force microscopy: unsaturated versus saturated molecular junctions, Journal Of Physical Chemistry B 106 (11), 28132816.

Zhang S, Xue F, Wu R, Cui J, Jiang Zm, Yang Xj (2009), Conductive Atomic Force Microscopy Studies On The Transformation Of Gesi Quantum Dots To Quantum Rings, Nanotechnology Apr $1 ; 20(13)$. 

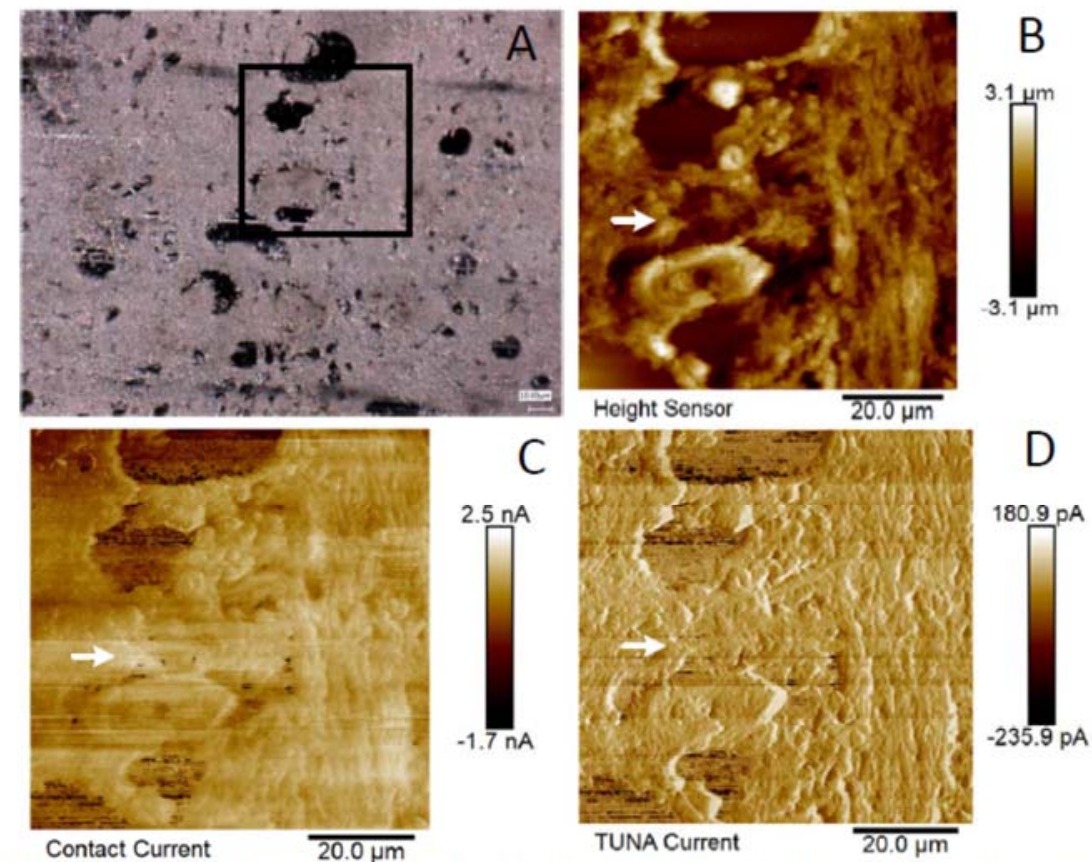

FIGURE 1A. A) Optical photograph of SN tissue on foil with $76 \mu \mathrm{m} \times 76 \mu \mathrm{m}$ inspection area in box. B) AFM scan showing height data relative to midpoint. C) Contact averaged current, autoscale ranging from -1.7 to $2.5 \mathrm{nA}$. D) cycle averaged current, autoscale ranging from -235.9 to 180.9 pA. Arrow shows location of detail scan.

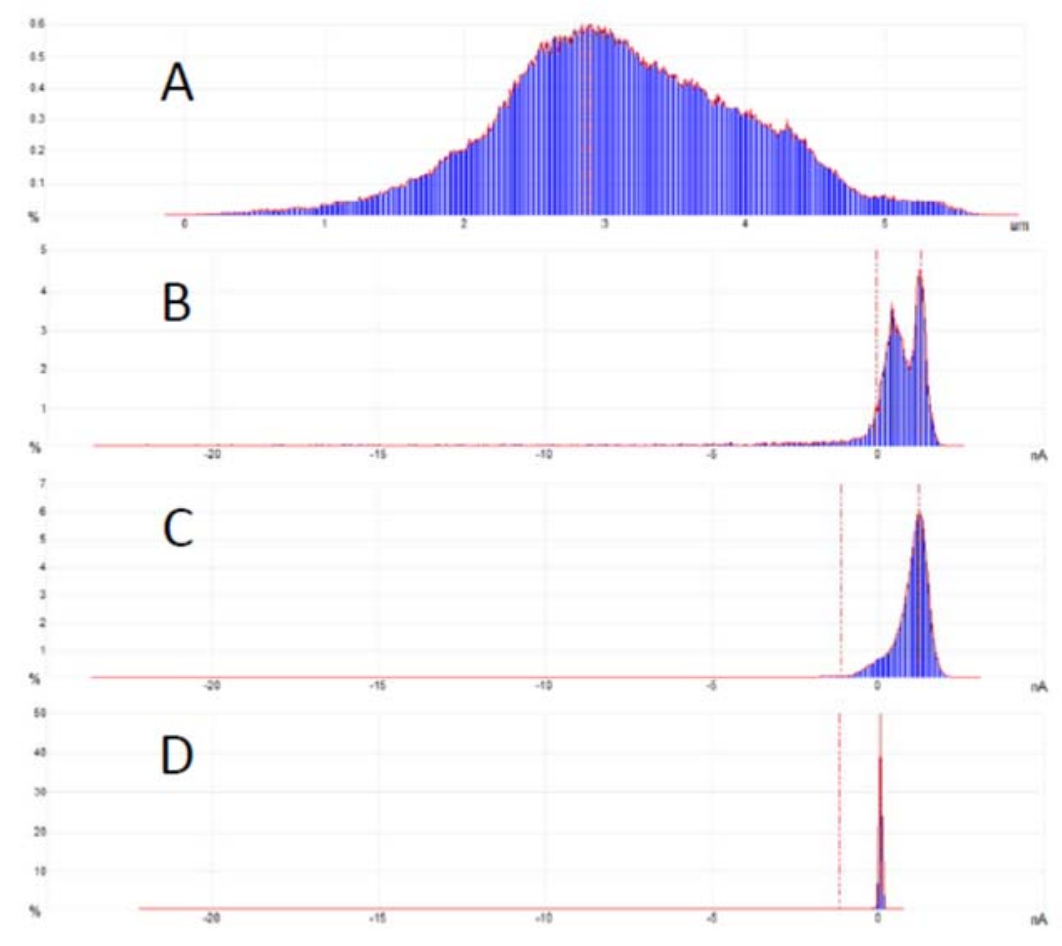

FIGURE 1B. A) AFM height data histogram. B) c-AFM contact averaged current histogram. C) c-AFM peak current histogram. D) c-AFM cycle average current histogram. 


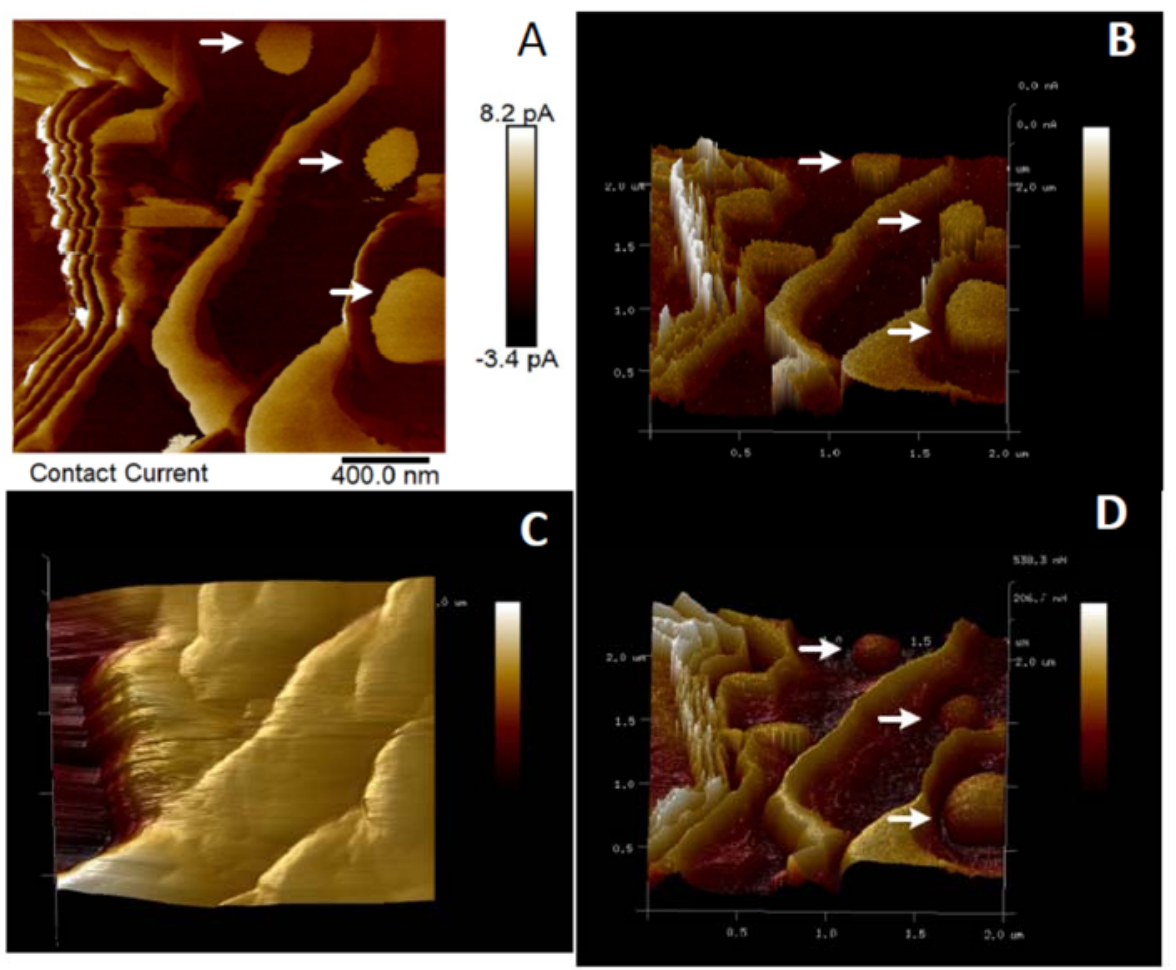

FIGURE 2A. A) 2D contact averaged current $2 \mu \mathrm{m} \times 2 \mu \mathrm{m}$ detail area, auto scale ranging from 3.4 to 8.2 pA. B) 3D perspective, contact averaged current, same scale. C) perspective, AFM scan showing height data, autoscale ranging from -2.3 to $1.2 \mu \mathrm{m}$ relative to midpoint. $\mathrm{D}$ ) perspective adhesion, autoscale ranging from -125 to $538 \mathrm{nN}$. Arrow shows location of neuromelanin structures.

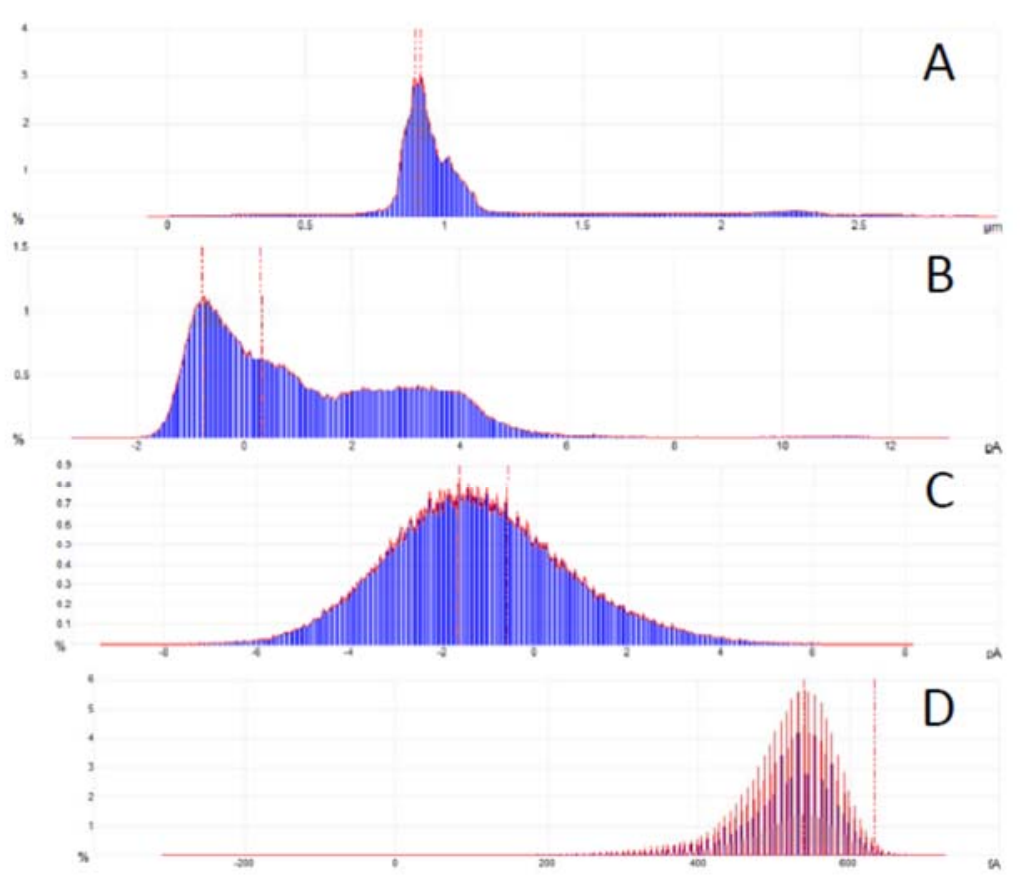

FIGURE 2B. A) AFM height data histogram. B) C-AFM contact averaged current histogram. C) cAFM peak current histogram. D) c-AFM cycle average current histogram. 

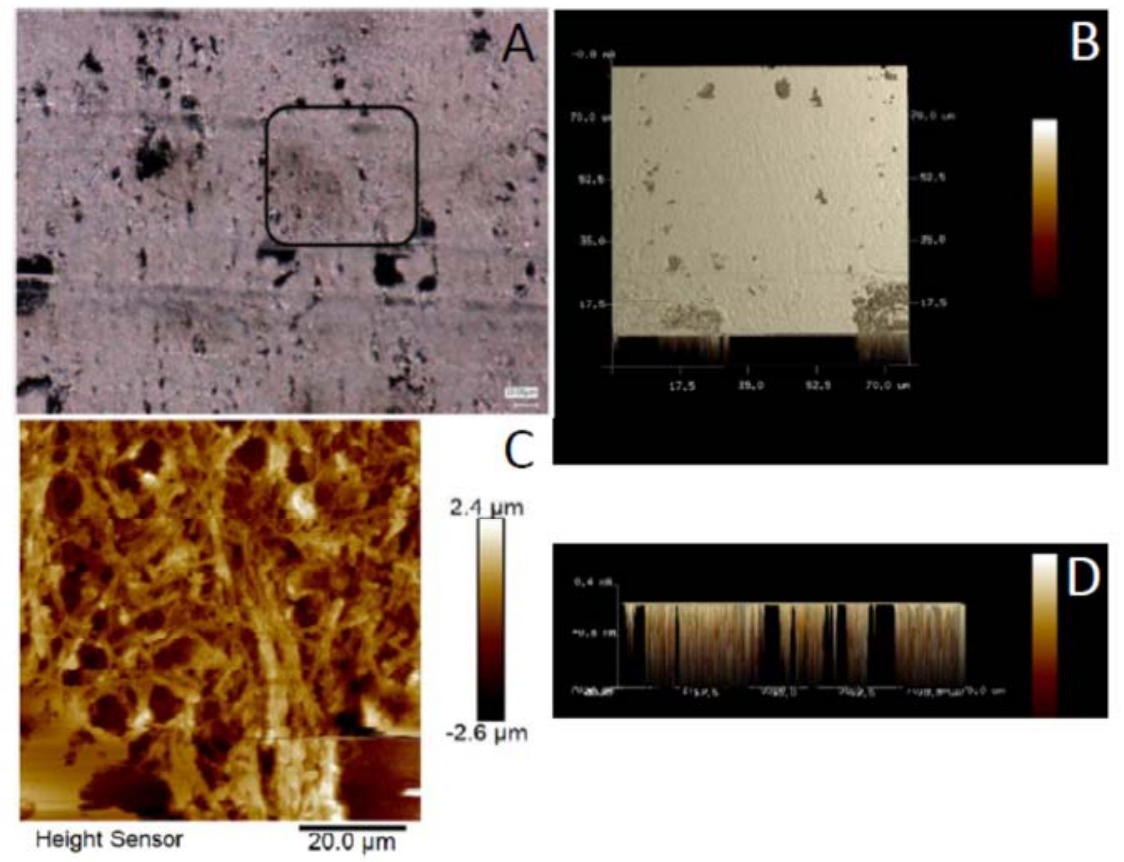

FIGURE 3A. A) Optical photograph of SN tissue on foil with $76 \mu \mathrm{m} \times 76 \mu \mathrm{m}$ inspection area in box. B) Contact averaged current, autoscale ranging from -2.1 to $0.4 \mathrm{nA}$. C) AFM scan showing height data relative to midpoint, autoscale -26 to $2.4 \mu \mathrm{m}$. D) Contact averaged current side view, showing absence of positive currents and mostly negative currents.

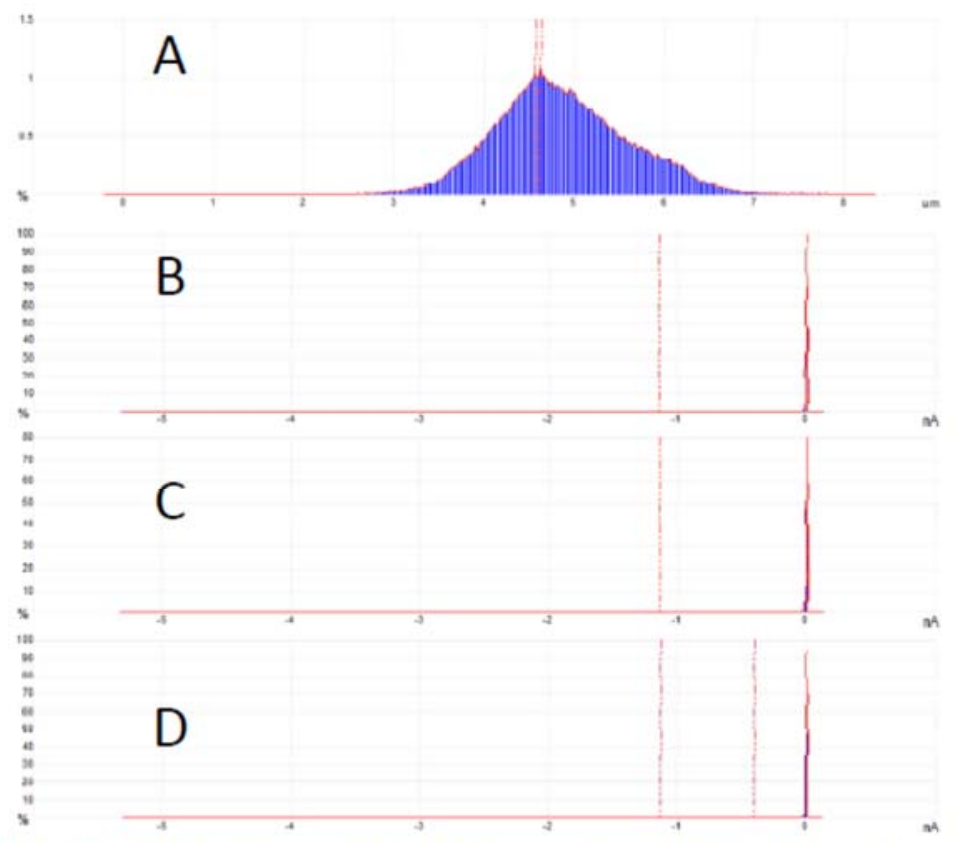

FIGURE 3B. A) AFM height data histogram. B) c-AFM contact averaged current histogram. C) c-AFM peak current histogram. D) c-AFM cycle average current histogram. 